\title{
AC 2010-308: A PLATFORM INDEPENDENT METHODOLOGY FOR TEACHING STUDENTS TO LEVERAGE THE POWER OF PARAMETRIC DESIGN TOOLS
}

\section{Michael Johnson, Texas A\&M University}

Johnson is an assistant professor in the Department of Engineering Technology and Industrial Distribution at Texas A\&M University. Prior to joining the faculty at Texas A\&M, he was a senior product development engineer at the 3M Corporate Research Laboratory in St. Paul, Minnesota for three years. He received his B.S. in mechanical engineering from Michigan State University and his S.M. and Ph.D. from the Massachusetts Institute of Technology. Johnson's research focuses on design tools, specifically, the cost modeling and analysis of product development and manufacturing systems; CAD methodology; and engineering education.

\section{Udaya Bhanu Prakash Sunku, Texas A\&M University}

Sunku is a graduate student in Mechanical Engineering at Texas A\&M University. He has a a Bachelors of Engineering degree in Mechanical Engineering from Osmania University, Hyderabad, India. 


\title{
A Platform Independent Methodology for Teaching Students to Leverage the Power of Parametric Design Tools
}

\begin{abstract}
One of the great contributions to engineering productivity over the last two decades has been the introduction of parametric computer-aided design (CAD) programs. In an increasingly competitive environment where more and more products have ever decreasing lifetimes, these programs allow engineers to make rapid design changes and quickly communicate designs to various customers and vendors. CAD has become an indispensable tool for most engineering professionals.

Too often CAD courses are taught as platform specific means to reach some geometric end. Conveying design intent and leveraging the parametric nature of these tools is not emphasized. This reduces the ability of students to produce designs which others can quickly change; the capacity of others to comprehend the organization of the model is also limited. This focus on a geometric end also causes students to concentrate on expedient platform specific means of reaching that end.
\end{abstract}

In this paper a methodology is detailed for teaching CAD in a manner that demonstrates the importance of design intent. The production of models that are clearly organized and easily changed is emphasized. Platform independent exercises which can be used to demonstrate and emphasize these goals are detailed. Data collected at the beginning and the end of the semester to assess the students' confidence with the parametric and feature-based nature of CAD are presented. Students showed a significant increase in their confidence and ability to describe parametric and feature-based concepts.

\section{Introduction}

Computer-aided design (CAD) tools have become indispensable for most engineering professionals. In an increasingly competitive global economy, there is expanding product variety and decreasing product lifetimes. ${ }^{1}$ Today's engineering and engineering technology students will enter a workplace where CAD is used throughout the development process ${ }^{2}$. CAD has become a valuable tool that allows engineers to quickly create and update designs and communicate them to others. The ability to use CAD is essential for most engineers today.

Most engineers will likely use several versions of CAD programs over their career. This may be due to the changes in their job, software changes at their company, or general software versioning (Pro/Engineer has had eight major versions in the past ten years). For their skills to remain in demand, it is essential that today's engineer be able to easily transfer knowledge to a new program version or platform. Unfortunately, CAD is often taught in a platform specific manner that shows students how to produce certain geometry with a specific CAD package. Students then focus on the expedient manner with which to produce that geometry. The exercises presented in this paper are designed to teach students lessons that apply generally to all higher-end parametric CAD programs. The focus is on helping students convey design intent using these programs. 
The design intent of a model should capture its function and allow the model to be easily changed $^{3-5}$. Design intent should be at the core of the CAD modeling education process. It encompasses feature ${ }^{\dagger}$ selection, order, and organization ${ }^{3}$. This will allow students to produce models that are amenable to change and use modern CAD tools to their full potential. Unfortunately, most CAD modeling instruction is focused on teaching declarative knowledge the key strokes and button picks required to perform particular tasks in certain CAD platforms ${ }^{6-}$

${ }^{8}$. Design intent would fall under the category of strategic knowledge ${ }^{9}$. This will allow them to apply the lessons learned in class in a new setting, when most likely they will be using a new version of the software or possibly a different CAD platform.

\section{Background}

The exercises described in this paper were prepared for a junior level design course in a mechanical and manufacturing engineering technology program. The CAD portion of the course is taught in a laboratory with each section comprising ten to fourteen students. The laboratory is one hour and fifty minutes long. The software used in the course is Pro/Engineer Wildfire 4.0. Each laboratory session consists of two parts. The first part is a guided exercise during which the students watch the instructor perform the steps required to create the desired geometry. The instructor's screen is projected and the students follow along, performing the same steps at their computers. During the second part, the students are given a representation (either drawing or rendering) of the final geometry that they are supposed to produce and offered assistance on an as needed basis. The exercises detailed in this paper were developed for use as guided exercises (first part of the laboratory session). These exercises usually required between thirty and fortyfive minutes to complete.

While the exercises were developed for use with Pro/Engineer, the authors have ensured that they are compatible with Solid Works. The authors are also confident that these exercises can be easily adapted to any higher-end CAD program without losing their content or educational value. The exercises presented below attempt to teach students some fundamental lessons about parametric design which will transcend both course and CAD program.

\section{Exercises}

\section{Exercise 1}

The goal of the first exercise is to demonstrate three main characteristics of higher-end CAD programs; the fact that they are feature-based, parametric, and provide solid models. ${ }^{4}$ This exercise is usually performed during the first session of the laboratory. The students are given graph paper and instructed to draw an isometric and three projection views of the part shown in Figure 1. Once the students have created the specified drawings, they are instructed to change the overall width of the box from four units to three and move the holes from one unit away from the edges to one-half unit from the edge. They then update all of their drawings.

\footnotetext{
${ }^{\dagger}$ Features are the building blocks of parametric CAD models. Depending on the software, they include basic elements (e.g., blocks, bosses, pockets, or holes) or sketch manipulations (e.g., extrusions, revolves, or blends).
} 


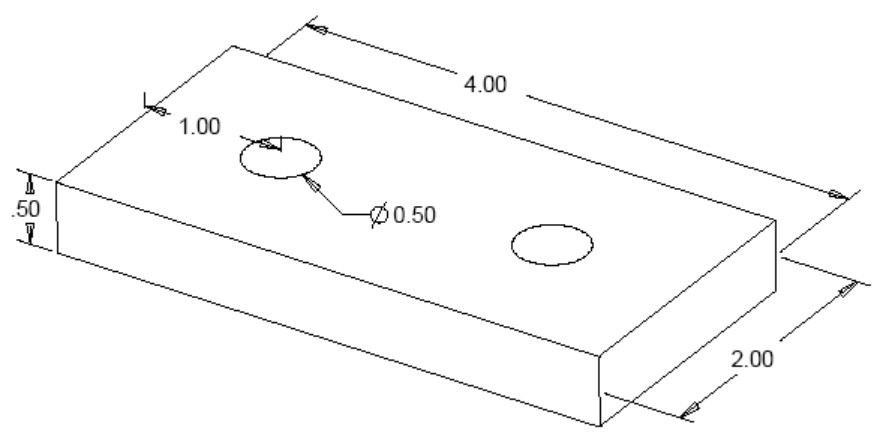

Figure 1. Representation of Box for Drawing Exercise

After the second set of drawings is completed, the CAD program is started and the instructor guides the students through the creation of the component they had initially hand drawn (free hand sketch). During the creation of the part, it is reinforced that each step involves a feature being added. Once the part is created, students are shown various projection, isometric, and section views of the part to emphasize its solid nature. An explanation of the component's use in finite element modeling simulations is given (it could also be demonstrated). Next the students are shown how to make a simple drawing of the part with the same isometric and projection views they had created previously on paper. Finally, the students are shown how to change the dimensions of their part and shown how the drawing updates with the new dimensions (see Figure 2). They are asked to compare this with their previous changes using the graph paper or analogous experiences with non-parametric drawing programs. The time and effort required to make changes is emphasized; a brief history of the progress from manual drafting to current CAD systems is also given. This simple exercise demonstrates some of the key capabilities (feature-based, parametric, and provides solid models) of CAD programs and can be easily adapted to most platforms.

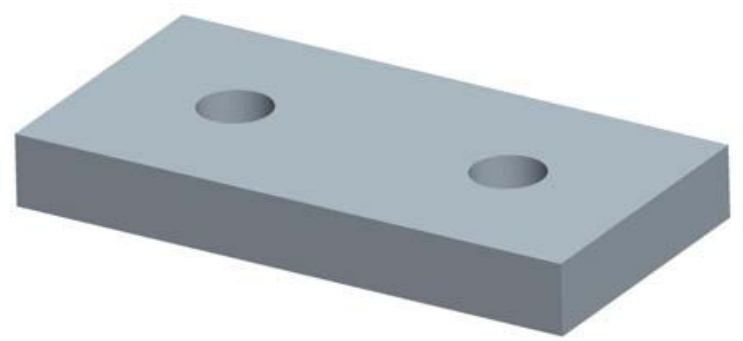

a.

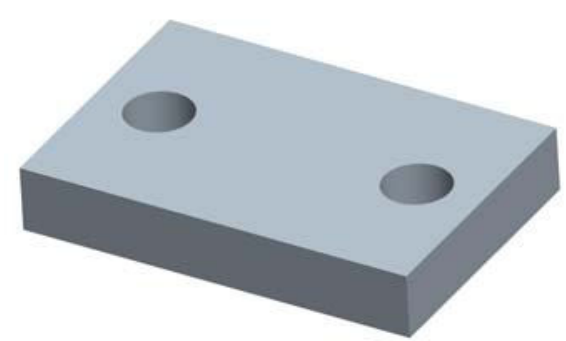

b.

Figure 2. a.) Original Part; b.) Altered Part.

\section{Exercise 2}

The goal of the second exercise is to introduce students to relations and reference geometry, and explain their use in relaying design intent. The students are told to draw a simple extruded box section. They are then instructed to put a through slot in the front of the box section; the slot is a specified vertical distance from the top of the box section and centered horizontally on the front face. Next, a second through slot is added to the box section. It is specified that this through slot is equal in width to that of the previous slot. Relations are used to specify that the distance from the bottom of the first to the top of the second slot is equal to the distance from the top of the first 
slot to the top of the box section. Additional features are then added to the part (e.g. rounds chamfers, see Figure 3a). After the initial part is completed (Figure 3a), the students are shown how to update the placement and width of the top slot changing only two dimensions. The part is then updated (see Figure 3b). The instructor then explains that if the intent of the part is to have slots that are spaced equally and have equal widths, this should be conveyed in the design. The connection between clear design intent and simple updating is emphasized.

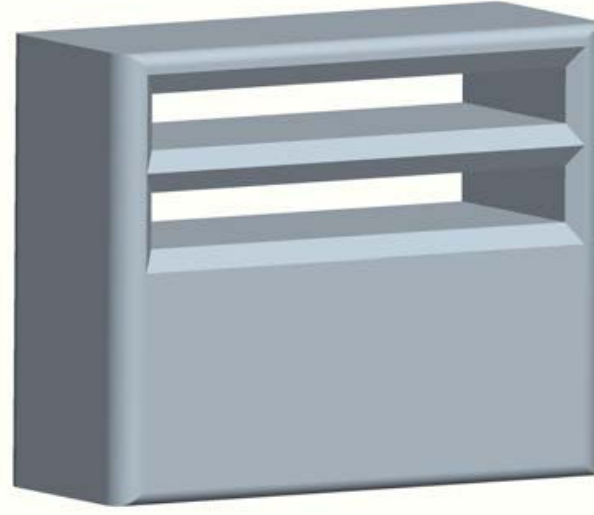

a.

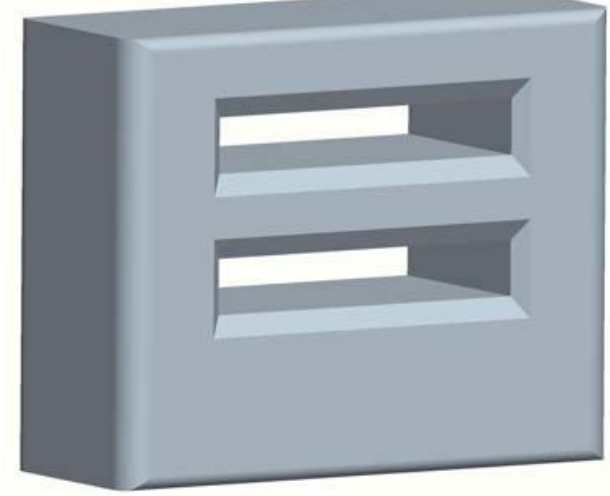

b.

Figure 3. Two box sections: a.) prior to change; b.) after change.

\section{Exercise 3}

The goal of the third exercise is to convey the importance of properly organizing and referencing geometry to convey the proper design intent. This exercise is adapted from a similar example in Toogood (p.5-2). ${ }^{4}$ In this exercise the students start with an extruded rectangular section (see Figure 4a). They are told that the part contains two through holes located a specified distance apart and centered with respect to the short edge of the block (see Figure 4c). Before putting the holes into the rectangular section, a full round is added to one end of the part (see Figure 4b). The first hole is added using the full round as a placement reference. The second hole is added using datum planes (either default or previously created) as references. Next the students are instructed to change the full round to a chamfer (see Figure 4d). When the students attempt this, an error occurs (either they will have to delete the hole or are unable to delete the round, depending on CAD platform). Students are then told this is an example of the importance of placing features in the proper order and relaying the proper design intent. The intent of the design is that the holes are a specified distance apart and centered with respect to the short edge of the part. Having the first hole related to full round does not convey this, while it constrains the ability to change that aspect of the part without changing one of the holes. Next, they are told that important geometry (the hole pattern) should be placed early in a design and related to the most primitive features available. Next the students are shown how to relate the second hole to datum planes as was the case with the first hole. Finally they add the chamfer back onto the part and are shown the ease with which the chamfer can be changed to a round and vice versa. 


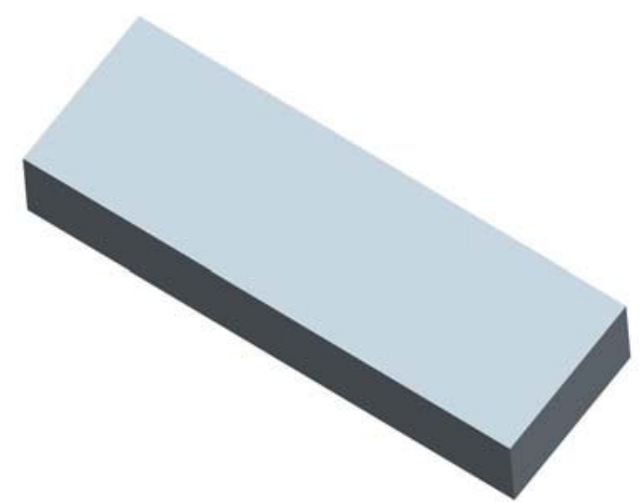

a.

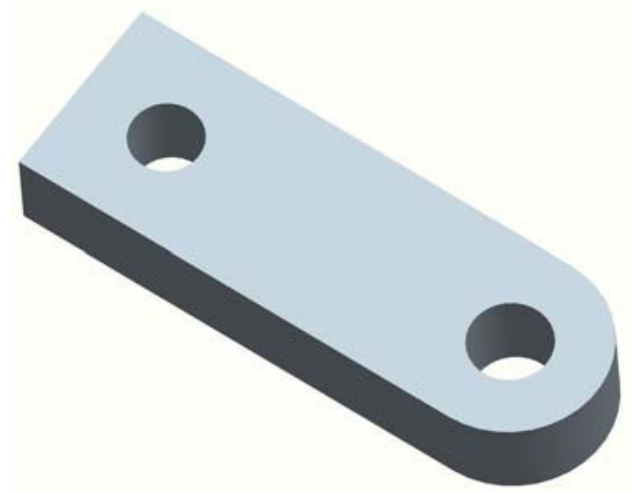

c.

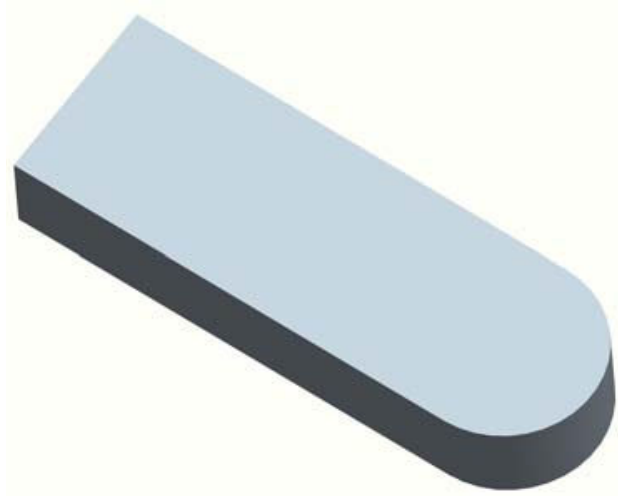

b.

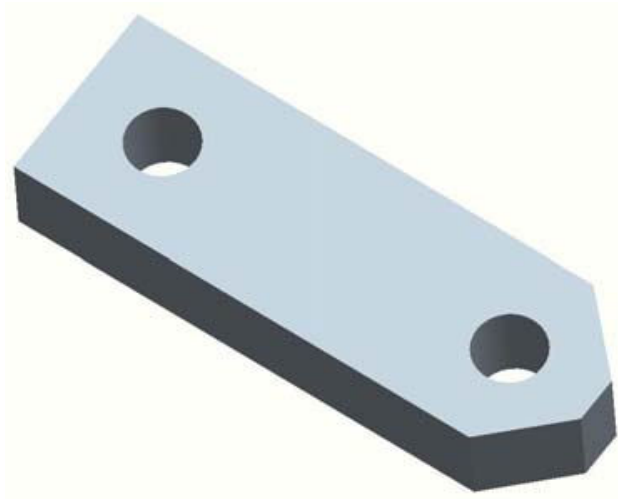

d.

Figure 4. Various Stages of Designed Block Section with Holes a) preliminary section; b) section with round; c) section with round and holes; d) section with chamfer and holes.

\section{Exercise 4}

The final exercise further emphasizes the importance of design intent, though here, in relation to assemblies. The students are instructed to make a plate with a through hole in the center and four small through holes in the corners (see Figure 5a). The four corner holes are created using a pattern. Relations are then used to make the holes an equal horizontal and vertical distance from the center of the top surface of the plate. The plate is then used as the first part of a new assembly. Four blocks and four bolts are then added to the assembly using the corner holes as placement constraints (see Figure 5b). The students are then instructed to change the two dimensions for the placement of the first corner hole in the plate. This updates the entire assembly and maintains the desired symmetry. The ease with which this change is made is pointed out. The importance of designing parts that update as expected when changed is emphasized It is also noted that this is especially important when those parts are used in assemblies. 


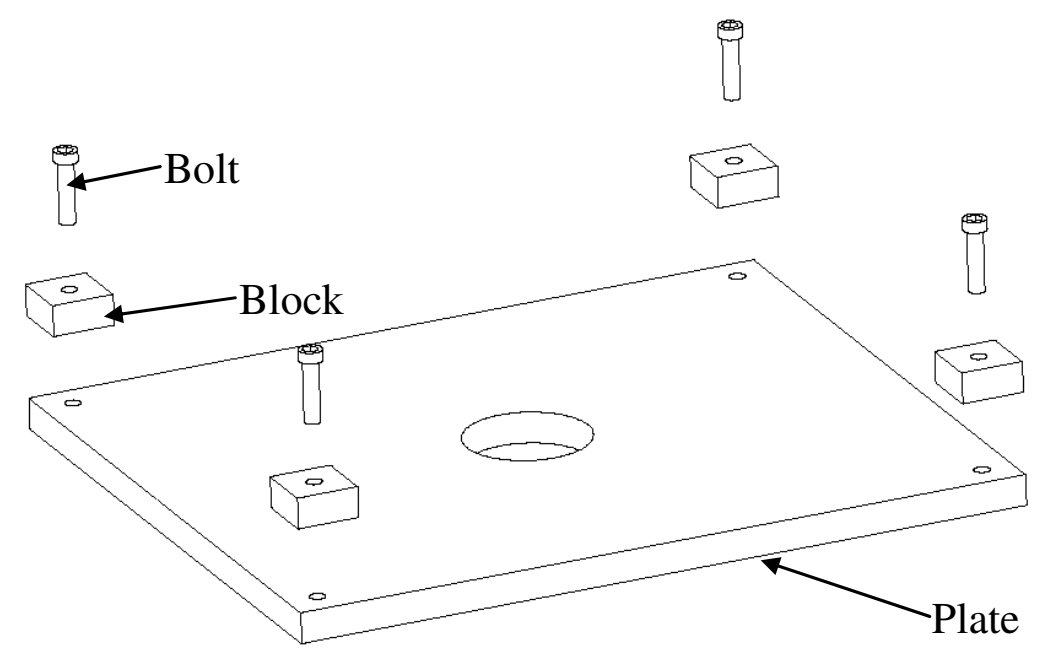

a.

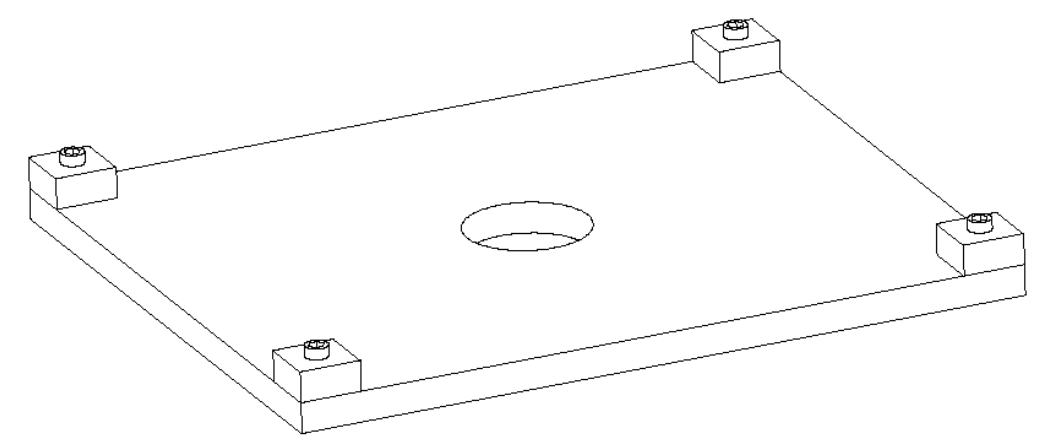

b.

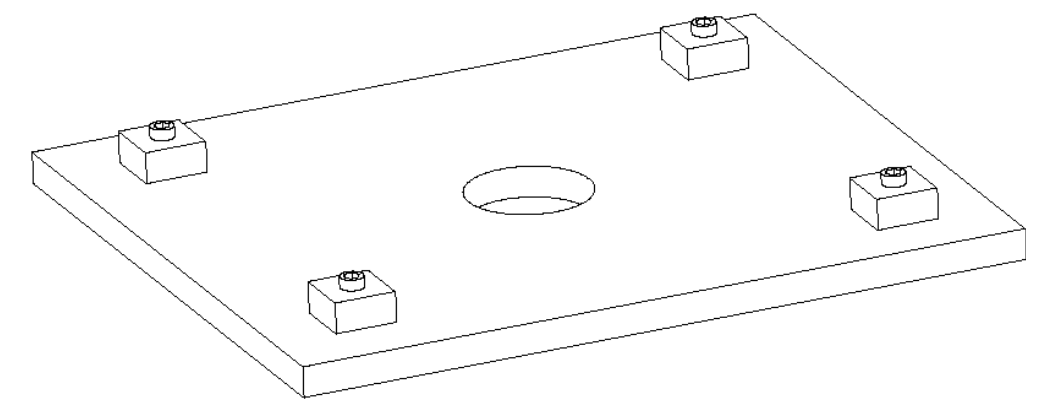

c.

Figure 5. Assembly of Parts Using Easily Changed Plate a) exploded view; b) initial design; c) altered design.

\section{Empirical Results}

While direct data regarding the students' comprehension of design intent was not captured (this was deemed too ambiguous a concept to assess at the beginning of the course), questions regarding the parametric and feature-based nature of CAD were used as proxies. Since these attributes of CAD are essential to properly conveying design intent, students' understanding of them provides a marker for their wider understanding of design intent. This questions were asked at the beginning of the laboratory portion of the course (before Exercise 1) and at the end. Data regarding the $\mathrm{CAD}$ packages that students had previously used and their confidence with their 
skill level using these packages were also collected. A copy of the survey taken at the beginning and end of the course is shown in the appendix (the CAD previous experience questions were omitted at the end of the course). Data for two recent semesters are shown in Table 1 (parametric and feature-based question data) and Table 2 (previous CAD experience). The beginning of the course data collected contained 54 points; the end of course data contained 49 (this was due to course attrition and absences).

Table 1. Survey Data

\begin{tabular}{|l|c|c|c|c|}
\hline & $\begin{array}{c}\text { Beginning } \\
\text { of Course }\end{array}$ & $\begin{array}{c}\text { End of } \\
\text { Course }\end{array}$ & T-Stat & $\begin{array}{c}\text { Significance } \\
\text { (one-tail) }\end{array}$ \\
\hline Feature-based Confidence & 2.7 & 4.9 & -7.09 & 0.000 \\
\hline Feature-based Correct & $25 \%$ & $78 \%$ & -6.36 & 0.000 \\
\hline Parametric Confidence & 2.2 & 4.3 & -8.88 & 0.000 \\
\hline Parametric Correct & $15 \%$ & $63 \%$ & -8.09 & 0.000 \\
\hline Placing Features Correct & $87 \%$ & $100 \%$ & -2.81 & 0.003 \\
\hline Part Change Correct & $91 \%$ & $98 \%$ & -1.61 & 0.055 \\
\hline Hole Pattern Correct & $96 \%$ & $100 \%$ & -1.43 & 0.080 \\
\hline
\end{tabular}

Table 2. Previous Experience Data

\begin{tabular}{|l|c|c|}
\hline Experience & $\begin{array}{c}\text { Percent } \\
\text { Reporting } \\
\text { Experience }\end{array}$ & $\begin{array}{c}\text { Average } \\
\text { Confidence }\end{array}$ \\
\hline UGS & $0 \%$ & N/A \\
\hline SolidWorks & $72 \%$ & 4.6 \\
\hline Inventor & $22 \%$ & 4.7 \\
\hline ProlEngineer & $4 \%$ & 4.5 \\
\hline CATIA & $0 \%$ & N/A \\
\hline AutoCAD & $52 \%$ & 4.8 \\
\hline
\end{tabular}

The confidence of the students to correctly define "feature-based" and "parametric" with relation to CAD was statistically significantly improved from the beginning of the course to the end of the course. The confidence rating improved over two points (on a seven point scale) in each case. The ability of the students to define these terms correctly was also significantly improved. The percentage correctly defining these terms increased from $25 \%$ to $78 \%$ in the case of "featurebased" and from $15 \%$ to $63 \%$ in the case of "parametric". As this might be seen as a result of any experience with a higher-end CAD program, the data regarding students' previous experience with CAD were also examined. Over $85 \%$ of the students had some previous experience with higher-end CAD programs (those listed in Table 2, save AutoCAD). The average confidence with these programs was 4.4. The other multiple choice questions asked in the survey also showed improvements in the number of students correctly answering. Students had significant experience with feature-based and parametric solid modeling tools, but did not realize what these concepts meant.

\section{Conclusions}

A set of platform independent exercises to teach the main benefits of higher-end parametric CAD programs is presented. Students are shown the main benefits of parametric CAD programs in contrast to lower-end drawing programs (i.e. they are feature-based, parametric, and provide solid models). These exercises show the importance of properly ordering and referencing 
features. The benefits of properly conveying design intent and its effect on the ability to make rapid and desired changes is also shown. The authors have tested these exercises with two major CAD programs and it is expected that they can be easily adapted to many others. These exercises allow students to learn lessons that go beyond learning expedient methods to produce geometry for a particular CAD program.

The exercises and focus of this course resulted in an improved ability and confidence of the students to define the "feature-based" and "parametric" nature of higher-end CAD programs. Students' ability to answer questions related to best-practice modeling methods also improved. These proxies for the understanding of design intent all improved after the course. This resulted in spite of the students having significant experience and confidence in using other higher-end CAD tools prior to their experiences in this course.

\section{Bibliography}

1. D. A. Field, "Education and Training for CAD in the Auto Industry," Computer-Aided Design 36 (14), 14311437 (2004).

2. S. D. Eppinger and A. R. Chitkara, "The New Practice of Global Product Development," MIT Sloan Management Review 47 (4), 22-30 (2006).

3. B. Caldwell and G. M. Mocko, "Ttite," in ASME 2008 International Design Engineering Technical Conferences \& Computers and Information in Engineering Conferences (Brooklyn, New York, 2008), pp. Paper No. DETC2008-50015.

4. A. Rynne, W.F. Gaughran, and B. McNamara, "Ttite," in 17th International Conference on Production Research (Blacksburg, VA, 2003).

5. R. Toogood, Pro Engineer Wildfire 2.0 Tutorial and Multimedia CD. (SDC Publications, Edmonton, 2004).

6. L.G. Lamit, Pro/Engineer wildfire 4.0. (Cengage Learning, Mason, OH, 2008).

7. R. F. Hamade, H. A. Artail, and M. Y. Jaber, "Evaluating the Learning Process of Mechanical CAD Students," Computers \& Education 49 (3), 640-661 (2007).

8. Z. Ye, W. Peng, Z. Chen, and Y-Y. Cai, "Today's Students, Tomorrow's Engineers: an Industrial Perspective on CAD Education," Computer-Aided Design 36 (14), 1451-1460 (2004).

9. I. Chester, "Teaching for CAD Expertise," International Journal of Technology and Design Education 17 (1), 23-35 (2007). 


\section{Appendix}

What other CAD programs have you used (Check all that apply)?

Unigraphics/UGS___ SolidWorks

ProlEngineer CATIA

Other(Please Name)

How confident are you in your abilities with this program (7-Very Confident/Expert; 1- Not Confident/Really Novice)

Program 1 (Name)___ Confidence

Program 2 (Name) Confidence

How confident are you in your ability to define what feature-based means with respect to CAD? (7-Very Confident; 1- Not Confident)

Define feature-based with respect to CAD

How confident are you in your ability to define what parametric means with respect to CAD? (7-Very Confident; 1- Not Confident)

Define parametric with respect to CAD

\section{Multiple Choice (Circle One Answer)}

When placing a feature in $\mathrm{CAD}$ is it better to reference:
A. A primitive feature that is unlikely to change
B. An unrelated, but close/recently added feature
C. I have no idea

When designing a part that will probably change, is it better to:
A. Design a few really complex features
B. Several simple features
C. I have no idea

If you wanted a four-hole pattern to be symmetric in a part, that best would be accomplished by:
A. Placing four individual holes in the proper location
B. Setting up a pattern with a mathematical relationship
C. I don't know 